\title{
AUTOMATED EXTRACTION OF 3-D GROUND CONTROL POINTS FROM SAR IMAGES - AN UPCOMING NOVEL DATA PRODUCT
}

\author{
Ulrich Balss ${ }^{1}$, Hartmut Runge ${ }^{1}$, Steffen Suchandt ${ }^{1}$, Xiao Ying Cong ${ }^{2}$ \\ ${ }^{1}$ Remote Sensing Technology Institute (IMF), German Aerospace Center (DLR), \\ D-82230 Oberpfaffenhofen, Germany \\ ${ }^{2}$ Remote Sensing Technology Chair (LMF), Technische Universität München (TUM), \\ D-80333 Munich, Germany
}

\begin{abstract}
This paper deals with a new Synthetic Aperture Radar (SAR) processing system for the generation of 3-D Ground Control Points with geodetic accuracy. With his help, laborious in situ measurements using Global Navigation Satellite System (GNSS) receivers can be replaced by remote sensing methods. It is shown how suitable point targets are extracted from the SAR images in an automated process and how its 3-D coordinates can be obtained by the Stereo SAR approach. The accuracy achieved was determined by means of GNSS reference measurements in two sample scenes. The measured accuracy is at decimeter level for north, east and height.
\end{abstract}

Index Terms - Synthetic aperture radar, imaging geodesy, ground control points

\section{INTRODUCTION}

There is a wide variety of measurement methods for accurate 3-D capture of the environs which can deliver a high relative accuracy but only insufficiently provide absolute coordinates with respect to a global reference frame. A possibility to overcome this problem is the use of Ground Control Points (GCPs) with known absolute 3-D coordinates.

A frequently used mean to capture precise absolute 3-D coordinates are in situ measurements based on the Global Navigation Satellite System (GNSS). But often, a terrestrial geodetic survey is not practical or even impossible: The journey for the staff may be too costly or the region inaccessible or too dangerous. We therefore developed a Synthetic Aperture Radar (SAR) based remote sensing method by which GCPs can be gathered around the globe without the need to enter the region.

The 3-D models to be ortho-rectified may stem from Laser Scanner (LiDAR) and stereo cameras. The sensor may be mounted on vehicles, aircrafts, satellites and lately also drones. The main applications are cartography and surveying. Prerequisite for the transfer of GCPs on the target data is an unambiguous identification of the GCPs in SAR image and target data. In the process, we take advantage of the fact that metallic poles, mounted on a flat surface, (e.g. lamp poles or traffic signs) constitute radar reflectors that occur as ideally focused points in a SAR image. The measured coordinates represent the base point on the radar illuminated side of the pole.

\section{GEODETIC SAR}

The prevalent application areas of space-borne SAR are weather and time of day independent observations of the earth surface. However, several recent studies [1-4] revealed that SAR offers as well the ability for high precision measurement of absolute positions.

In particular, accuracy at the centimeter level is proven for the German SAR satellites TerraSAR-X (TSX-1) and TanDEM-X (TDX-1) [4]. One prerequisite for this accuracy is a thorough design of all subsystems including sensor hardware, instrument calibration, orbit determination, and in SAR data processing the elimination of several processor approximations. The other prerequisite is the precise correction of the radar time coordinates (azimuth and range) for all signal propagation delays and for geodynamic effects like plate tectonics and solid earth tides [5][6].

\section{POINT TARGET EXTRACTION AND LOCALIZATION}

In particular in urban areas, SAR images acquired by stateof-the-art high resolution SAR sensors like TerraSAR-X or COSMO-SkyMed show an myriad of point-like objects which are however of different value for the exploitation as GCPs. The majority of them stems from building structures like protrusions or window cutouts. Often, such structures have complex radar reflection properties and it is hard to assign their phase center position to an actual, single object. When occurring in clusters, even the assignability of corresponding point scatterers in different SAR images is a cumbersome and hardly automatable task.

In contrast, poles of lamps or traffic signs show rather simple reflection properties. There is a double bounce be- 
tween the front edge of the pole and the ground (which ideally is the asphaltic road surface). The phase center of the radar echo, which yields the GCP, corresponds to the base point of the pole. Many of them can be found in urban areas and along of roads and motorways. In particular, corresponding observations of sole free-standing lamp poles or traffic signs are easily assignable because they cannot be confused with nearby similar objects.

Thus, our GCP extraction processor focuses on such isolated point scatterers. Bright point-like objects are detected in SAR images, their positions are measured at subpixel accuracy level and the target characteristics are analyzed. The subpixel position of the target is converted to radar times on base of the SAR product annotation and corrected for signal propagation delays and geodynamic effects. Peak width and signal to clutter ratio (SCR) constitute selection criteria in order to discard inappropriate targets. Based on both parameters also an estimate for the expected localization accuracy is computed according to Stein's equation [7][8].

The determination of geodetic 3-D coordinates by Stereo SAR [9-11] requires the usage of at least two SAR images that are acquired with different view angle. From the geometrical point of view, an angular difference of 90 degrees, which can be realized by combining acquisitions from one ascending and one descending orbit, would be optimum. However, one hardly finds point targets which are flawlessly visible over such a high angular range and even if that was the case, the object almost always has significantly different phase centers in both acquisitions. In the end, the typical angular difference for our application amounts to approx. 10-20 degree which results for acquisitions from two or three spatially consecutive, unidirectional (i.e. only ascending or only descending) orbits.

The matching of presumably corresponding point scatterers in the analyzed SAR images is applied in an automated process on base of a coarse geolocation of the scatterers using a DEM. However, the accuracy of typical up to now available DEMs does not suffice to provide decimeter level localization accuracy in one step. Thus, the precise geodetic coordinates of the found point targets result not until the subsequent Stereo SAR processing step.

As a byproduct, the Stereo SAR approach as well provides an estimate for the localization accuracy of each obtained GCP. This error measure as well yields a good consistency check whether the observed point scatterers refer to the same object. Here, the availability of additional datatakes beyond the minimum configuration of two acquisitions is of advantage.

\section{LOCALIZATION RESULTS}

Output format for the localization result of our GCP processor is a Keyhole Markup Language (KML) file with extended data. In this way, the output data can be conveniently

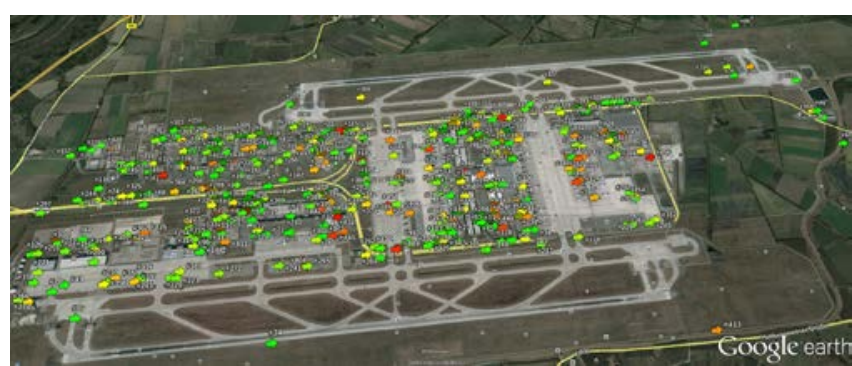

Figure 1: GCPs from the Munich airport test site (overview)

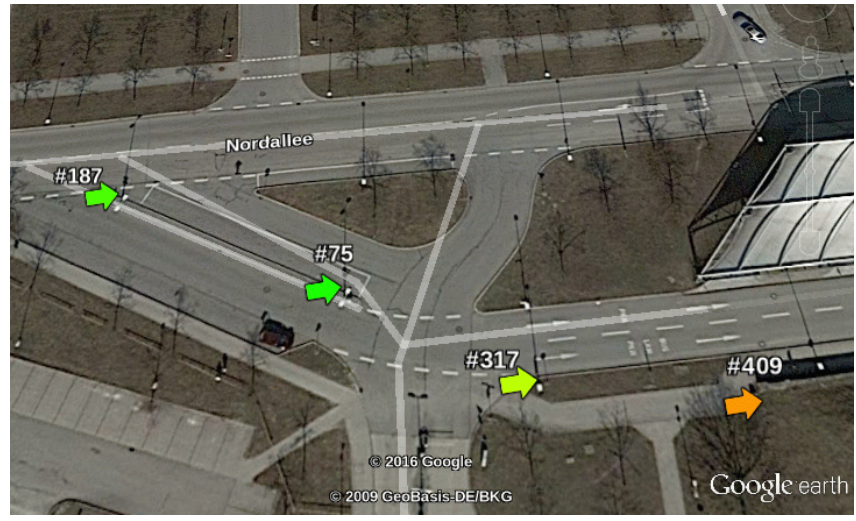

Figure 2: GCPs from the Munich airport test site (close-up)

visualized and analyzed using GoogleEarth ${ }^{\mathrm{TM}}$. The obtained GCPs are ordered and numbered by ascending expected localization error. The chosen place mark style in our GCP product is a colored arrow pointing to the respective GCP. Its orientation reflects the mean illumination direction in the underlying SAR images. The color codes the estimated localization error, varying from green via chartreuse, yellow and orange to red where green means the best possible, red the worst just acceptable quality.

Additional KML tags for auxiliary data hold information about top level acquisition and quality parameters of each GCP and are listed in a separate sub-window when clicking on the respective place mark. There are:

- the precise 3-D coordinates of the GCP annotated in the International Terrestrial Reference Frame 2008 (ITRF2008) in two representations

○ as WGS84 ellipsoid (latitude, longitude, height) coordinates

- as Cartesian (X, Y, Z) coordinates

- the reference epoch these coordinates refer to (this is important because virtually any point on earth moves due to plate tectonics)

- the estimated localization error in range, azimuth and elevation (which are the principal axes of the error ellipsoid obtained by Stereo SAR) and

- unit vectors reflecting the orientation of the principal axes of the error ellipsoid w.r.t. the ITRF2008 coordinate system. 
Figure 1 (full scene) and Figure 2 (close-up) gives an impression on the visualization of our GCP product in GoogleEarth $^{\mathrm{TM}}$. The processed sample scene is located at Munich airport (MUC). The close-up shows roads and a part of the parking lot. The color coded place marks in Figure 2 demonstrate how the expected localization accuracy depends on the direction of radar illumination: Where the lamp pole is illuminated face toward the road, double bonce via lamp pole and the smooth road surface result in a high radar backscatter and consequently good expected localization accuracy (object \#75 and \#187, green arrows). Illuminated face away from the road, double bunce is via lamp pole and meadow or bare soil leading to a lower radar backscatter and inferior localization accuracy (object \#409, orange arrow). In Figure 2, one sees as well that the positions of the place marks fit well to the base points of lamp poles in the optical image. However, the here observed correspondence rather reflects the accurate positioning of the GoogleEarth ${ }^{\mathrm{TM}}$ image in this particular scene but it is no instrument to verify the localization quality of our SAR derived GCPs.

\section{VERIFICATION}

In order to verify the localization accuracy of our automated extracted GCPs, we initially chose two test sites: Bad Kötzting and Oberpfaffenhofen, both in Germany, for the following test procedure. On the one hand side, we acquired TerraSAR-X High Resolution Spotlight (HS) and Staring Spotlight (ST) datatakes of these test sites, each test site in two or three acquisition geometries (i.e. from two or three neighboring orbits) and obtained a set of GCPs by our GCP extraction processor. On the other hand side, we commissioned a surveying company to measure precise reference coordinates for some lamp poles and traffic signs by GNSS based terrestrial survey. Due to the required effort, the acquisition of reference coordinates by terrestrial geodetic survey has to be restricted to a small subset of the automatically extracted GCPs.

Figure 3 shows some of the test objects at the Bad Kötzting site. At all, terrestrial reference coordinates for 12 lamp poles, 4 traffic signs and one wooden power line pole were available. The Stereo SAR based GCPs were computed either on base of 59 TerraSAR-X HS datatakes from three descending orbits or 22 datatakes from two ascending orbits. It is a piece of luck that two Stereo SAR stacks already exist from this test site. However, the huge number of SAR images is not mandatory for the applicability of our GCP extraction processor. Table 1 comprises the measured offset between Stereo SAR and GNSS coordinates for these targets. For all targets in analysis, the measured offset in north, east or height direction is below or near to $20 \mathrm{~cm}$. The standard deviation of the offset amounts to about $7-10 \mathrm{~cm}$ in north, east and height direction. However, we observe an average offset at the centimeter level. Whether this offset is due to systematic effects, is subject of ongoing investigations.

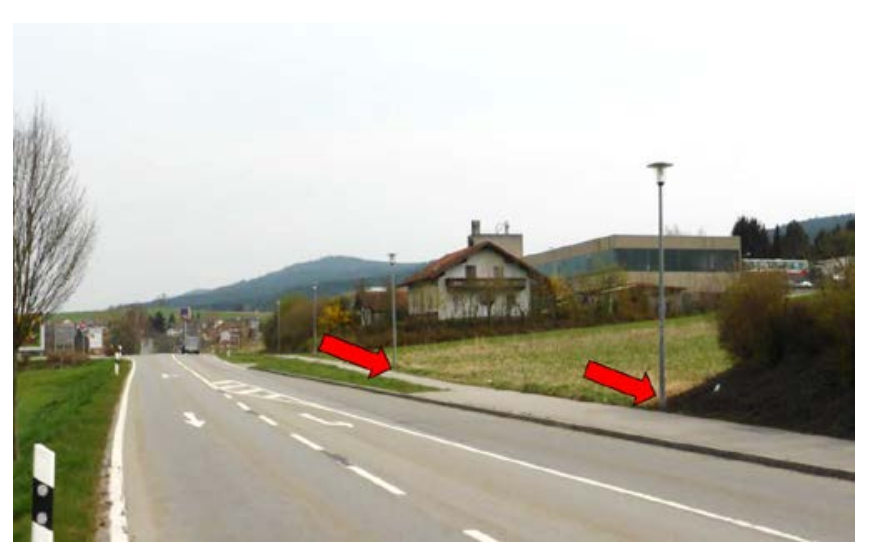

Figure 3: Some of the lamp poles at the Bad Kötzting test site (Photo by Vermessungsbuero Patrzek)

A small offset between the expected phase center position (at the base point of the pole) of the scatterers and the true position might be caused by the backscatter of the mounted lamp housing or traffic signs. Also the effect of a convex or inclined road surface on the double bounce backscatter geometry has to be investigated. At the aspired accuracy level of few decimeters or even centimeters, as well the accuracy of the reference coordinate acquisition has to be verified. In a first step, we crosschecked the utilized GNSS receiver (Leica) against a competitive product (Trimble) and observed consistency of the acquired coordinates down to $1 \mathrm{~cm}$. Currently, we verify the GNSS coordinate ac-

Table 1: Verification results from the Bad Kötzting test site

\begin{tabular}{|l|r|r|r|}
\hline \multirow{4}{*}{ Target } & \multicolumn{3}{|c|}{ Difference Stereo SAR - GNSS [m] } \\
\cline { 2 - 4 } & North & East & Height \\
\hline lamp pole 1 & -0.015 & -0.022 & -0.018 \\
\hline lamp pole 2 & -0.099 & 0.065 & -0.068 \\
\hline lamp pole 3 & -0.108 & 0.070 & -0.074 \\
\hline lamp pole 4 & -0.096 & 0.076 & -0.105 \\
\hline lamp pole 5 & -0.120 & 0.042 & -0.125 \\
\hline lamp pole 6 & -0.166 & 0.040 & -0.073 \\
\hline lamp pole 7 & -0.138 & 0.070 & -0.107 \\
\hline traffic sign 1 & -0.019 & 0.035 & -0.200 \\
\hline lamp pole 8 & -0.068 & -0.098 & -0.183 \\
\hline lamp pole 9 & -0.200 & -0.085 & 0.046 \\
\hline lamp pole 10 & -0.064 & 0.048 & -0.219 \\
\hline traffic sign 2 & -0.153 & 0.219 & -0.209 \\
\hline \multicolumn{5}{|c|}{ Ascending } \\
\hline lamp pole 11 & 0.033 & 0.026 & 0.050 \\
\hline lamp pole 12 & -0.001 & 0.044 & -0.101 \\
\hline wooden pole & -0.071 & 0.077 & 0.052 \\
\hline traffic sign 3 & 0.080 & 0.087 & -0.074 \\
\hline traffic sign 4 & 0.221 & 0.025 & 0.021 \\
\hline \multicolumn{5}{|c|}{ Statistics } \\
\hline Mean & $\mathbf{- 0 . 0 5 8}$ & $\mathbf{0 . 0 4 2}$ & $\mathbf{- 0 . 0 8 2}$ \\
\hline Stddev. & $\mathbf{0 . 1 0 3}$ & $\mathbf{0 . 0 7 0}$ & $\mathbf{0 . 0 9 0}$ \\
\hline RMS & $\mathbf{0 . 1 1 8}$ & $\mathbf{0 . 0 8 2}$ & $\mathbf{0 . 1 2 1}$ \\
\hline
\end{tabular}


Table 2: Verification results from the Oberpfaffenhofen test site

\begin{tabular}{|l|r|r|r|}
\hline \multirow{2}{*}{ Target } & \multicolumn{3}{|c|}{ Difference Stereo SAR - GNSS [m] } \\
\cline { 2 - 4 } & North & East & Height \\
\hline \multicolumn{4}{|c|}{ Descending } \\
\hline lamp pole 1 & 0.015 & 0.129 & -0.155 \\
\hline lamp pole 2 & 0.014 & 0.179 & -0.102 \\
\hline lamp pole 3 & -0.106 & 0.144 & -0.158 \\
\hline lamp pole 4 & -0.008 & 0.205 & -0.230 \\
\hline \multicolumn{4}{|c|}{ Ascending } \\
\hline lamp pole 5 & -0.084 & 0.079 & -0.015 \\
\hline lamp pole 2 & -0.103 & 0.017 & 0.057 \\
\hline lamp pole 6 & 0.083 & 0.172 & 0.095 \\
\hline lamp pole 1 & -0.068 & 0.141 & 0.155 \\
\hline lamp pole 7 & -0.097 & 0.212 & 0.139 \\
\hline lamp pole 8 & -0.076 & 0.181 & 0.316 \\
\hline \multicolumn{5}{|c|}{ Statistics } \\
\hline Mean & $\mathbf{- 0 . 0 4 3}$ & $\mathbf{0 . 1 4 6}$ & $\mathbf{0 . 0 1 0}$ \\
\hline Stddev. & $\mathbf{0 . 0 6 5}$ & $\mathbf{0 . 0 6 0}$ & $\mathbf{0 . 1 7 2}$ \\
\hline RMS & $\mathbf{0 . 0 7 8}$ & $\mathbf{0 . 1 5 8}$ & $\mathbf{0 . 1 7 3}$ \\
\hline
\end{tabular}

quisition on base of EUREF (European Geodetic Reference Systems) station points with very precisely known reference coordinates, located at the geodetic observatory Wettzell, Germany.

At the Oberpfaffenhofen test site, the comparison of Stereo SAR based and GNSS based 3-D coordinates was performed on base of 8 lamp poles, where 4 poles are observable from ascending TerraSAR-X orbits, 2 from descending orbits and 2 from both orbit directions. 4 TerraSAR-X ST datatakes were acquired: Two ascending and two descending. In two separate processing runs, at each time GCPs from datatakes of equal orbit direction were extracted. Table 2 shows the verification result. Despite of the much lower number of used SAR images compared to the Bad Kötzting test site, the root mean square of the observed offsets between Stereo SAR and GNSS based coordinates is still below $20 \mathrm{~cm}$ in north, east and height direction.

Verification measurements at further test sites, situated also outside Germany, are subject of ongoing work.

\section{CONCLUSIONS AND OUTLOOK}

For urban areas with a high density of suitable objects like lamp poles the new SAR processing system can replace GNSS techniques for the determination of the geodetic position of Ground Control Points. It has been shown that with repeated measurements the same level of accuracy can be obtained. The main advantage over GNSS is the remote sensing approach which avoids the necessity to be on site for the measurement. Furthermore the process is completely automated and a whole ground control point net, e. g. of a city can be obtained with a minimum configuration of only two satellite acquisitions and the subsequent data processing. An operational data product will become available during the year 2016.

\section{ACKNOWLEFGEMENTS}

This work was partly funded by the Helmholtz Validation Funds (HVF) and by DLR's Technology Marketing department. The surveying was performed by Vermessungsbuero Patrzek (Nuremberg, Germany).

\section{REFERENCES}

[1] A. Schubert, M. Jehle, D. Small, and E. Meier, "Mitigation of Atmospheric Pertubations and Solid Earth Movements in a TerraSAR-X Time-Series," Journal of Geodesy, vol. 86, pp. 257-270, 2011.

[2] M. Eineder, C. Minet, P. Steigenberger, X.Y. Cong, and T. Fritz, "Imaging Geodesy - toward Centimeter-Level Ranging Accuracy with TerraSAR-X," IEEE Trans. Geosc. Remote Sens., vol. 49, pp. 661-671, 2011.

[3] A. Schubert, D. Small, M. Jehle, and E. Meier, "COSMOSkyMed, TerraSAR-X, and Radarsat-2 Geolocation Accuracy after Compensation for Earth-System Effects," Proc. IGARSS 2012, Munich, Germany, pp. 3301-3304, 2012.

[4] U. Balss, C. Gisinger, X.Y. Cong, R. Brcic, S. Hackel, and M. Eineder, "Precise Measurements of the Absolute Localization Accuracy of TerraSAR-X on the Base of Far-Distributed Test Sites," Proc. EUSAR 2014, Berlin, Germany, pp. 993-996, 2014.

[5] Petit, G., and B. Luzum, Eds., IERS Conventions (2010), Verlag des Bundesamtes für Kartographie und Geodäsie, 2010. [Online]. http://tai.bipm.org/iers/conv2010/conv2010.html.

[6] M. Eineder, U. Balss, S. Suchandt, C. Gisinger, X.Y. Cong, and H. Runge, "A Definition of Next-Generation SAR Products for Geodetic Applications," Proc. IGARSS 2015, Milan, Italy, pp. 1638-1641, 2015.

[7] S. Stein, "Algorithms for Ambiguity Function Processing," IEEE Trans. Accoust., Speech, Signal Process., vol. ASSP-29, pp. 588-599, 1981.

[8] R. Bamler, and M. Eineder, "Accuracy of Differential Shift Estimation by Correlation and Split-Bandwidth Interferometry for Wideband and Delta-k SAR Systems," IEEE Geosc. And Remote Sens. Letters, vol. 2, no. 2, pp. 151-155, 2005.

[9] P.H. Chen, and I.J. Dowman, "A Weighted Least Squares Solution for Space Intersection of Spaceborne Stereo SAR Data," IEEE Trans. Geosc. Remote Sens., vol. 39, no. 2, pp. 233-240, 2001.

[10] E. Sansosti, "A Simple and Exact Solution for the Interferometric and Stereo SAR Geolocation Problem," IEEE Trans. Geosc. Remote Sens., vol. 42, no. 8, pp. 1625-1634, 2004.

[11] C. Gisinger, U. Balss, R. Pail, X.X. Zhu, S. Montaseri, S. Gernhardt, and M. Eineder, "Precise Three-Dimensional Stereo Localization of Corner Reflectors and Persistent Scatterers with TerraSAR-X," IEEE Trans. Geosc. Remote Sens., vol. 53, no. 4, pp. 1782-1802, 2015. 